\title{
Some results for the weak convergence of semi-implicit split-step methods
}

\author{
Burhaneddin Izgi and Berivan Ari \\ Istanbul Technical University, Department of Mathematics, Istanbul, Turkey \\ Received: 15 August 2018, Accepted: 7 February 2019 \\ Published online: 6 March 2019.
}

\begin{abstract}
In this paper, we obtain some results for the weak convergence of semi-implicit split-step (SISS) methods which are recently developed to solve a class of nonlinear stochastic differential equation with non-Lipschitz drift term. First, we present some moment estimates based on the actual and numerical solutions of stochastic Ginzburg-Landau equations by SISS methods. Then, we show that our theoretical results are consistent with the numerical results that are obtained by performing simulations. Finally, we present the weak convergence rate of SISS methods is approximately 1 with respect to the numerical results.
\end{abstract}

Keywords: Split-step methods, semi-implicit split-step method, weak convergence.

\section{Introduction}

Recently, there are some crucial improvements for the numerical solution of nonlinear stochastic differential equations of the form

$$
d X_{t}=a\left(X_{t}\right) d t+\sigma\left(X_{t}\right) d W_{t} ; 0 \leq t \leq T, X(0)=X_{0} .
$$

with non or locally Lipschitz drift term in the literature. It is known that numerical solutions of this kind of stochastic differential equations with the explicit methods may explode in finite time [1]. Therefore, some of the scientists focus on the implicit or semi implicit methods to ensure the stability and consistency of the numerical solutions. For example, Tamed-Euler, Truncated Euler, split-step backward Euler, and semi-implicit split-step (SISS) methods are some of these methods, among others.

The tamed Euler scheme, which is a modified version of the Euler-Maruyama method, is introduced by Hutzenthaler et al. [2]. Then, it is alternative versions are developed by Sabanis, and Hutzenthaler et al. in [3] and [4], respectively. For the weak convergence results and some other extensions of this method, one may see [4] and the references therein. Another modified version of the Euler-Maruyama method is called the truncated Euler method. It is suitable for SDEs with superlinear drift terms under some constraints [5,6].

The split-step backward Euler (SSBE) method, which is exhibited by Mattingly et al in [7], preserves the ergodicity properties of a class of monotone nonlinear SDEs. Although it has good precisions and is useful for obtaining the moment bounds of the numerical approximations [8], it may not be good enough in terms of computation time since some nonlinear scalar/vector equations should be solved numerically at each step. Moreover, in 2006, Schurz discussed a class of implicit and partially implicit methods in his paper [9]. Additionally, Mao and Szpruch analyzed the strong convergence and stability properties of some implicit numerical schemes in [10]. 
Furthermore, Izgi and Cetin first introduced the semi-implicit split-step (SISS) methods and they showed some moment results of SISS methods for the scalar case in [11]. In 2018, they also developed and investigated the high dimensional versions of SISS methods for nonlinear SDEs with non-Lipschitz drift terms in [12]. After that, in [13], they presented Milstein type semi-implicit split step (MSISS) methods for nonlinear SDE with locally Lipschitz drift terms. They also work on strong convergence analysis of the SISS and MSISS methods in [14] and [15], respectively. According to our the literature review, there is no study about the weak convergence analysis of these new methods yet. Therefore, we study the weak convergence of the semi-implicit split-step methods in this paper. We especially focus on SISS1 and SISS3 methods among others and obtain some results for the first moment of these numerical methods. We also obtain some moment bounds for the actual solution of the stochastic Ginzburg-Landau equation. Finally, we achieve to show that the weak convergence rate of these methods is approximately 1 by performing the simulations for the Ginzburg-Landau equation. The numerical analysis results also confirm the consistency of the moment bounds for the actual and numerical solutions of the equation obtained by SISS1 and SISS3 methods. In addition to these, one may see [16] for the second moment boundaries and simulation results.

The remainder of the paper is organized as follows. The semi-implicit split-step (SISS) methods are represented in this section. We present some theorems for the moment estimates of the SISS1 and SISS3 methods and actual solution of the stochastic Ginzburg-Landau equation and prove them in Section 2. In Secton 3, we perform simulations with the scalar version of Ginzburg-Landau SDE and exhibit the partial weak convergence analysis results. The paper concludes in Section 4.

\subsection{Semi implicit split-step methods for Ginzburg-Landau equation}

We consider the scalar version of the generalized stochastic Ginzburg-Landau equation

$$
d X(t)=\left(A X(t)-\delta X^{r}(t)\right) d t+\sigma(X(t)) d W(t), 0<t \leq T
$$

for an odd positive integer $r \geq 3$, and constants $\delta>0, A \in \mathbb{R}$ with $X(0)=x_{0}$, where $\sigma($.$) satisfies the linear growth$ condition. Then, we know that the first step of the split-step backward Euler method [8] for this scalar SDE reduces to

$$
y=x+\Delta\left(A y-\delta y^{r}\right)
$$

On the other hand, Izgi and Cetin presented the approximation for the drift term $a(y)=A y-\delta y^{r}$ with $\rho_{1}(x, y)=A y-$ $\delta y x^{r-1}$, or with $\rho_{2}(x, y)=A x-\delta y x^{r-1}$ in $[11,12]$ when they introduced SISS method. If we solve $y=x+\rho_{1}(x, y) \Delta$ and $y=x+\rho_{2}(x, y) \Delta$ for $y$, respectively, then we obtain the following expressions

$$
f^{\Delta}(x)=\frac{x}{1+\Delta\left(\delta x^{r-1}-A\right)} \text { while } a^{\Delta}(x)=\frac{a(x)}{1-\Delta\left(A-\delta x^{r-1}\right)}
$$

and

$$
g^{\Delta}(x)=\frac{(1+A \Delta) x}{1+\Delta \delta x^{r-1}} \text { while } a^{\Delta}(x)=\frac{a(x)}{1+\Delta \delta x^{r-1}}
$$

where $f^{\Delta}(x)=x+\Delta a^{\Delta}(x)$ and $g^{\Delta}(x)=x+\Delta a^{\Delta}(x)$ with the corresponding $a^{\Delta}(x)$.

By using the above approximations, the four SISS methods, which are introduced by Izgi and Cetin [11,12], are in the following forms for a generalized version of the stochastic Ginzburg-Landau equation: 
The first SISS method (SISS1): For $f^{\Delta}(x)=\frac{x}{1+\Delta\left(\delta x^{r-1}-A\right)}$

$$
X_{k+1}=f^{\Delta}\left(X_{k}\right)+\sigma\left(X_{k}\right) \Delta W_{k+1},
$$

The second SISS method (SISS2): For the same $f^{\Delta}(x)$ above and $\sigma^{\Delta}(x)=\sigma\left(f^{\Delta}(x)\right)$,

$$
X_{k+1}=f^{\Delta}\left(X_{k}\right)+\sigma^{\Delta}\left(X_{k}\right) \Delta W_{k+1} .
$$

The third SISS method (SISS3): For $g^{\Delta}(x)=\frac{(1+A \Delta) x}{1+\Delta \delta x^{r-1}}$,

$$
X_{k+1}=g^{\Delta}\left(X_{k}\right)+\sigma\left(X_{k}\right) \Delta W_{k+1} .
$$

The fourth SISS method (SISS4): For the same $g^{\Delta}(x)$ above,

$$
X_{k+1}=g^{\Delta}\left(X_{k}\right)+\sigma\left(g^{\Delta}\left(X_{k}\right)\right) \Delta W_{k+1} .
$$

where $\Delta=\Delta t=T / n$ and $\Delta W_{k+1}=W_{(k+1) \Delta t}-W_{k \Delta t}$ for each $k=0,1,2, \ldots n$.

Remark. By Lemma 1 and Theorem 1 in [12], we know that we have all moments of the actual solution, and all moments of the numerical solutions of equation (1) obtained by SISS methods exist for sufficiently small $\Delta$.

\section{Some moment bounds for the SISS Methods}

In this section, we obtain some moments bounds for the SISS methods based on the stochastic Ginzburg-Landau equation for $r=3$ while the diffusion term is $\sigma(X)=\sigma X$. We especially focus on SISS1 and SISS3 methods. Although the all moments of the numerical solutions need to be investigated for the weak convergence analysis of SISS methods, as a first step for this analysis, we work on the first moment bounds of these methods. Note that similar theoretical results may obtain easily for SISS2 and SISS4 methods using their relationship with SISS1 and SISS3 methods, respectively.

Theorem 1. The iterations $X_{k}$ using the first SISS method (2) to solve the equation (1) for $r=3$ and $\sigma(X)=\sigma X$ satisfy the following:

(i) The upper bound for the first moment of the iterations while $\delta>0, A \in \mathbb{R}$ is

$$
E\left[X_{k+1}\right] \leq \frac{X_{0}}{(1-A \Delta)^{k+1}}
$$

for all $k=0,1,2, \ldots, n$ and sufficiently small $0<\Delta \leq T$.

(ii) There is sufficiently small $\Delta_{0}>0$ such that $X_{0}<\sqrt{1+2 \sqrt{\frac{1-A \Delta_{0}}{\delta \Delta_{0}}}} \cdot\left(1-A \Delta_{0}\right)^{n}$ while $1-A \Delta_{0}>0$ then the lower bound for the first moment of the iterations

$$
E\left[X_{k+1}\right] \geq \frac{1}{(1-A \Delta)^{k+1}}\left(1+\sqrt{\frac{\delta \Delta}{1-A \Delta}}\right)^{-2(k+1)} X_{0}
$$

holds for all $k=0,1,2, \ldots, n$ and $0<\Delta \leq \Delta_{0}$ while $\delta>0, A \in \mathbb{R}$. 
Proof. (i) If we take the expectation of the iterations for the first SISS method $X_{k+1}=f^{\Delta}(X)+\sigma\left(X_{k}\right) \Delta W_{k+1}$ then we have $E\left[X_{k+1}\right]=E\left[f^{\Delta}\left(X_{k}\right)\right]$ using the fact that $\left\{\sigma\left(X_{k}\right) \Delta W_{k+1}=\sigma X_{k} \Delta W_{k+1}, k \geq 0\right\}$ is a martingale by above Remark, and basic stochastic calculus rules. Then, we have the following results after some iterations for $f^{\Delta}(x)=\frac{x}{1+\Delta\left(\delta x^{r-1}-A\right)}$ with $r=3$ which is given in (2) for equation (1).

$$
E\left[X_{k+1}\right]=E\left[\frac{X_{k}}{1-\left(A-\delta X_{k}^{2}\right) \Delta}\right] \leq \frac{E\left[X_{k}\right]}{1-A \Delta},
$$

then we have

$$
\begin{aligned}
E\left[X_{1}\right] \leq & \frac{X_{0}}{1-A \Delta} \\
E\left[X_{2}\right] \leq & \frac{E\left[X_{1}\right]}{1-A \Delta} \leq \frac{X_{0}}{(1-A \Delta)^{2}} . \\
& \vdots \\
E\left[X_{k+1}\right] \leq & \frac{X_{0}}{(1-A \Delta)^{k+1}} .
\end{aligned}
$$

(ii) If we use similar approaches in (i), then we have

$$
E\left[X_{k+1}\right]=E\left[f^{\Delta}\left(X_{k}\right)\right]=E\left[\frac{X_{k}}{1-\left(A-\delta X_{k}^{2}\right) \Delta}\right]=E\left[\frac{X_{k}}{u+m X_{k}^{2}}\right]
$$

where $u=1-A \Delta$ and $m=\delta \Delta$. Now, let's define $g(x)$ as $\frac{x}{u+m x^{2}}$, then

$$
\begin{aligned}
g(x) & =\frac{1}{u}\left(\frac{x}{1+\frac{m}{u} x^{2}}\right) ; \text { let } N=\frac{m}{u} \\
& \geq \frac{1}{u}\left(\frac{x}{(1+\sqrt{N})^{2}}\right) \text { while } x<\sqrt{1+2 \sqrt{\frac{1-A \Delta}{\delta \Delta}}}
\end{aligned}
$$

Thus, we have $g(x) \geq h(x)$ where $h(x)=\frac{1}{u}\left(\frac{x}{(1+\sqrt{N})^{2}}\right)$. By the condition on $X_{0}$ and the result in (i) above it is clear that the monotonicity $E\left[g\left(X_{k}\right)\right] \geq E\left[h\left(X_{k}\right)\right]$ holds for all $k$. Now if we use this fact in (4), then we have the following after some iterations:

$$
\begin{aligned}
E\left[X_{k+1}\right] & \geq \frac{1}{u}\left(\frac{1}{(1+\sqrt{N})^{2}}\right) E\left[X_{k}\right] \\
& \geq \frac{1}{u^{2}}\left(\frac{1}{(1+\sqrt{N})^{4}}\right) E\left[X_{k-1}\right] \\
& \vdots \\
& \geq \frac{1}{u^{k+1}}\left(\frac{1}{(1+\sqrt{N})^{2(k+1)}}\right) X_{0} \\
& =\left(\frac{1}{(1-A \Delta)^{k+1}}\right)\left(1+\sqrt{\frac{\delta \Delta}{1-A \Delta}}\right)^{-2(k+1)} X_{0}
\end{aligned}
$$

Theorem 2. The iterations $X_{k}$ using the third SISS method (3) to solve the equation (1) for $r=3$ and $\sigma(X)=\sigma X$ satisfy the following: 
(i) The upper bound for the first moment of the iterations while $\delta>0, A \in \mathbb{R}$ is

$$
E\left[X_{k+1}\right] \leq \frac{(1+A \Delta)^{k+1}}{1+\delta \Delta X_{0}^{2}} X_{0}
$$

for all $k=0,1,2, \ldots, n$ and sufficiently small $0<\Delta \leq T$.

(ii) For sufficiently small $\Delta_{0}>0$, if $X_{0}<\sqrt{1+\frac{2}{\sqrt{\delta \Delta_{0}}}} \cdot\left(1-A \Delta_{0}\right)^{-n}$ then the lower bound for the first moment of the iterations

$$
E\left[X_{k+1}\right] \geq \frac{(1+A \Delta)^{k+1}}{(\sqrt{\delta \Delta}+1)^{2(k+1)}} X_{0}
$$

holds for all $k=0,1,2, \ldots, n$ and $0<\Delta \leq \Delta_{0}$ while $\delta>0, A \in \mathbb{R}$.

Proof. (i) The proof can be done by using the similar steps that are used in the proof of Theorem 1 for the third SISS method $X_{k+1}=g^{\Delta}(X)+\sigma X_{k} \Delta W_{k+1}$ while $g^{\Delta}(x)=\frac{(1+A \Delta) x}{1+\Delta \delta x^{r-1}}$ with $r=3$. Then we have

$$
E\left[X_{k+1}\right]=E\left[g^{\Delta}\left(X_{k}\right)\right]=E\left[\frac{(1+A \Delta) X_{k}}{1+\Delta \delta X_{k}^{2}}\right]
$$

After some iterations, we have

$$
\begin{gathered}
E\left[X_{1}\right]=\frac{X_{0}(1+A \Delta)}{1+\delta \Delta X_{0}^{2}} \\
E\left[X_{2}\right] \leq(1+A \Delta) E\left[X_{1}\right]=(1+A \Delta)^{2} \frac{X_{0}}{1+\delta \Delta X_{0}^{2}} . \\
\vdots \\
E\left[X_{k+1}\right] \leq(1+A \Delta)^{k+1} \frac{X_{0}}{1+\delta \Delta X_{0}^{2}}
\end{gathered}
$$

(ii) It is clear that we have the following for SISS3 method

$$
E\left[X_{k+1}\right]=E\left[g^{\Delta}\left(X_{k}\right)\right]=E\left[\frac{X_{k}}{1+\delta \Delta X_{k}^{2}}\right](1+A \Delta)=E\left[\frac{X_{k}}{1+m X_{k}^{2}}\right](1+A \Delta) .
$$

where $m=\delta \Delta$. In this step, if we define $h(x)$ as $\frac{x}{1+m x^{2}}$ then

$$
h(x)=\frac{x}{(\sqrt{m} x+1)^{2}-2 \sqrt{m} x} \geq \frac{x}{(\sqrt{m}+1)^{2}}=g(x) \text { while } x<\sqrt{1+\frac{2}{\sqrt{\delta \Delta}}} .
$$

Use the monotonicity of $h(x) \geq g(x)$ in (5) and iterate it, then we obtain

$$
E\left[X_{k+1}\right] \geq(1+A \Delta) \frac{E\left[X_{k}\right]}{(\sqrt{m}+1)^{2}} \geq \frac{(1+A \Delta)^{k+1}}{(\sqrt{m}+1)^{2(k+1)}} X_{0}=\frac{(1+A \Delta)^{k+1}}{(\sqrt{\delta \Delta}+1)^{2(k+1)}} X_{0}
$$


Corollary 1. The terminal value, $X_{n}$, satisfies $E\left[X_{n}\right] \leq X_{0} e^{A T}$ while using the SISS1 and SISS3 methods to solve the equation (1) for $r=3$ and $\sigma(X)=\sigma X$ when $\delta>0, A \in \mathbb{R}$ and sufficiently small $0<\Delta \leq T$.

Theorem 3. The actual solution of equation (1) for $r=3$ and $\sigma(X)=\sigma X$ is given in equation (9) has the following upper and lower boundaries:

$$
\begin{aligned}
& E[X(t)] \leq X_{0} e^{A t} \\
& E[X(t)] \geq \frac{X_{0} e^{\left(A-\frac{3}{2} \sigma^{2}\right) t}}{\sqrt{1+\frac{2 \delta X_{0}^{2}\left(e^{\left(2 A+\sigma^{2}\right) t}-1\right)}{2 A+\sigma^{2}}}}
\end{aligned}
$$

while the model parameters $\delta>0, A \in \mathbb{R}$ with $X(0)=X_{0}$.

Proof. If we take the expected value of the actual solution where $F(t)=e^{\left(\frac{1}{2} \sigma^{2}-A\right) t-\sigma W(t)}$ then we have the following for the upper bound of the actual solution:

$$
\begin{aligned}
E[X(t)] & =E\left[F^{-1}(t)\left\{x_{0}^{-2}+2 \delta \int_{0}^{t} F^{-2}(s) d s\right\}^{-1 / 2}\right] \\
& \leq E\left[F^{-1}(t)\right] X_{0}, \text { by the expectation of the geometric Brownian motions, } \\
& \leq X_{0} e^{A t}
\end{aligned}
$$

Similarly, we start with

$$
E[X(t)]=E\left[\frac{F^{-1}(t)}{\sqrt{x_{0}^{-2}+2 \delta \int_{0}^{t} F^{-2}(s) d s}}\right]
$$

then, by Jensen's inequality, we obtain

$$
E[X(t)] \geq \frac{X_{0} e^{\left(A-\frac{1}{2} \sigma^{2}\right) t}}{E\left[e^{-\sigma W(t)} \sqrt{1+2 \delta X_{0}^{2} \int_{0}^{t} F^{-2}(s) d s}\right]}
$$

Now, if we use Cauchy-Schwarz inequality with Fubini's theorem then we have

$$
E[X(t)] \geq \frac{X_{0} e^{\left(A-\frac{1}{2} \sigma^{2}\right) t}}{\sqrt{e^{\frac{4 \sigma^{2}}{2} t}\left\{1+2 \delta X_{0}^{2} \int_{0}^{t} E\left[e^{\left(2 A-\sigma^{2}\right) s+2 \sigma W(s)}\right] d s\right\}}}=\frac{X_{0} e^{\left(A-\frac{3}{2} \sigma^{2}\right) t}}{\sqrt{1+\frac{2 \delta X_{0}^{2}\left(e^{\left(2 A+\sigma^{2}\right) t}-1\right)}{2 A+\sigma^{2}}}}
$$

after some calculations.

Corollary 2. The expected value of the actual solution of equation (1) for $r=3$ and $\sigma(X)=\sigma X$ at the terminal time $E[X(T)]$ is bounded above by $X_{0} e^{A T}$. 


\section{Simulation results}

In this section, we perform simulations via SISS methods for the stochastic Ginzburg-Landau equation, whose explicit solution is known, and present some extensive numerical analysis results. Especially, we investigate the consistency of our theoretical results, which can be used at the further theoretical frameworks for the weak convergence analysis of these methods, obtained for SISS1 and SISS3 methods. Meanwhile, we analyze their empirical rates of weak convergence.

For a numerical example, we consider the following SDE, which is the stochastic Ginzburg-Landau equation for $r=3$ with multiplicative noise,

$$
d X(t)=\left(A X(t)-\delta X^{3}(t)\right) d t+\sigma X(t) d W(t)
$$

for $X(t)=x_{0}>0$ fixed, and $0 \leq t \leq T$. The explicit and unique solution of this equation satisfies the following a.s. positive process

$$
X(t)=F^{-1}(t)\left\{x_{0}^{-2}+2 \delta \int_{0}^{t} F^{-2}(s) d s\right\}^{-1 / 2}
$$

where $F(t)=e^{\left(\frac{1}{2} \sigma^{2}-A\right) t-\sigma W(t)}$, satisfying the $\operatorname{SDE} d F(t)=F\left(\sigma^{2}-A\right) d t-\sigma F d W(t)$ with $F(0)=1$. This can be shown easily and it's derivation can be done by applying the Ito's rule to $Y(t)=X(t) F(t)$ with $Y(0)=x_{0}$.

Moreover, we perform the repeated simulations with the $A=-1, \delta=0.1, \sigma=1$, and $x_{0}=5$ model parameters while the number of simulations is $N=10.000$. Figure 1 and Figure 2 show that the upper and lower boundaries obtained for SISS1 and SISS3 methods are consistent with the numerical solutions of the SDE in (8).

Furthermore, Figure 3 is generated by using the boundaries, which are given by (6) and (7) in Theorem 3, for the actual solution (9) of Ginzburg-Landau equation. The figures also confirm that these boundaries keep the behavior of the solution of stochastic Ginzburg-Landau equation. Another important interpretation of these results is one may use these boundaries to show the usual rate of weak convergence of these methods in the theoretical proof which is left for a future project in this paper.

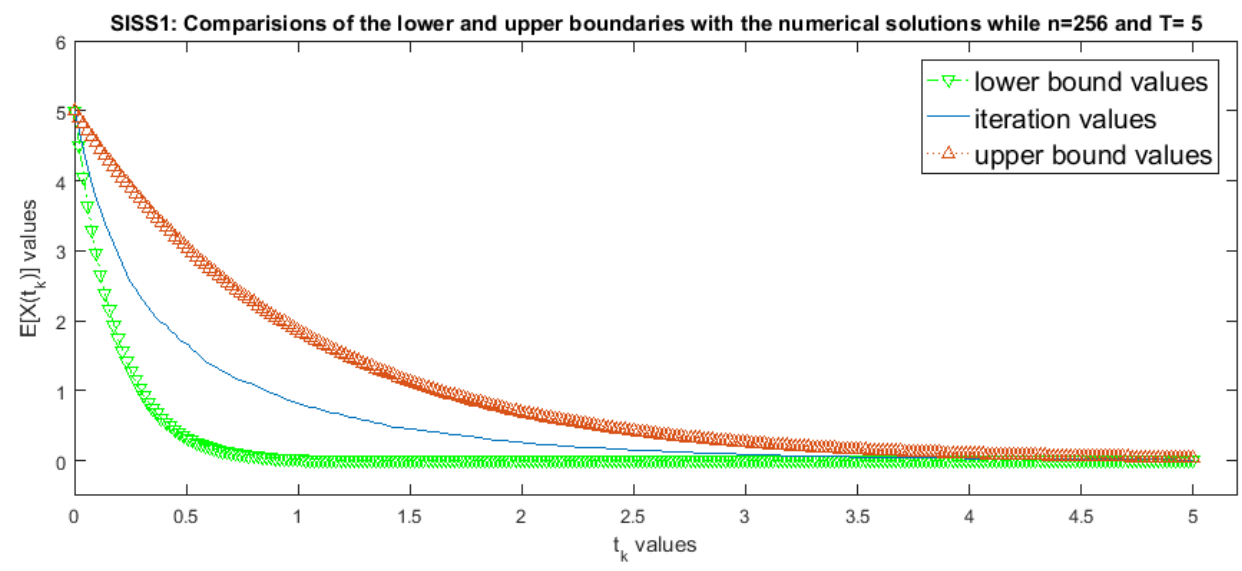

Fig. 1: SISS1: Comparisions of the boundaries with the numerical solutions. 


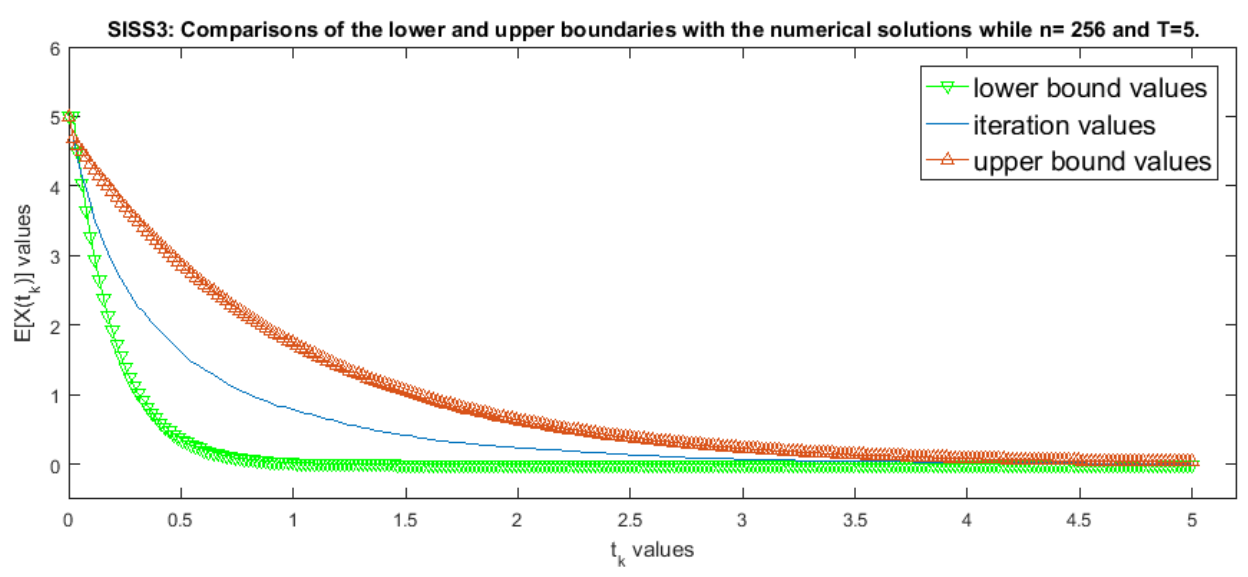

Fig. 2: SISS3: Comparisions of the boundaries with the numerical solutions.

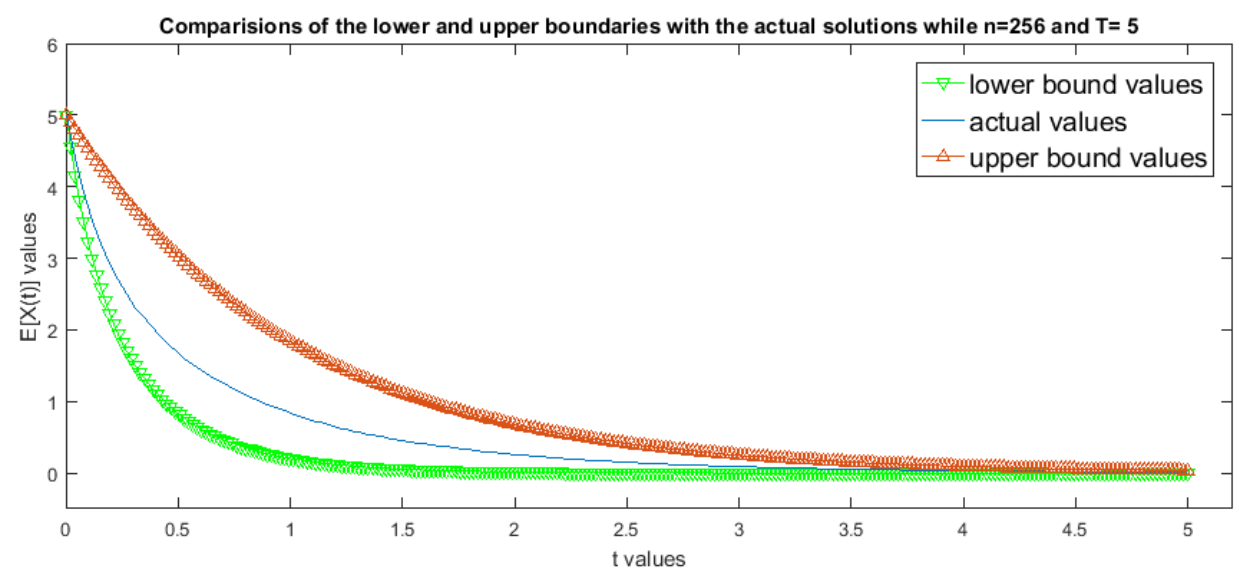

Fig. 3: Comparisions of the boundaries with the actual solutions.

On the other hand, it is sufficient to consider terminal time values for the weak convergence analysis. Therefore, we conduct repeated simulations of size $N=100.000$ for each method with the respective step size, $n=2^{6}, 2^{7}, 2^{8}$ and $2^{9}$, in order to investigate the rate of weak convergence of these methods empirically. Then, we show these analysis' results using the log-log graphs such that the comparisons of $E[X(T)]$ with $E\left[X\left(t_{n}\right)\right]$ and $E\left[X^{2}(T)\right]$ with $E\left[X^{2}\left(t_{n}\right)\right]$ are given in Figure 4 and Figure 5, respectively. Both figures suggest that the SISS1 and SISS3 achieve the usual weak convergence rate of 1. Moreover, we obtain similar results in Figures 4 and 5 for the higher moment values (i.e. $E\left[X^{3}(T)\right], E\left[X^{4}(T)\right]$ etc.), too.

\section{Conclusions}

We provide some partial theoretical and numerical results for the weak convergence analysis of the semi-implicit split-step (SISS) methods based on stochastic Ginzburg-Landau equation under some conditions of the model parameters. First, we find the first moment bounds for the SISS1 and SISS3 methods and the actual solution of the equation. Then, we perform simulations and succeed to show that the weak convergence rate of SISS methods is approximately 1 , as it is expected, with respect to the log-log graphs. Finally, we observe that our repeated simulation 


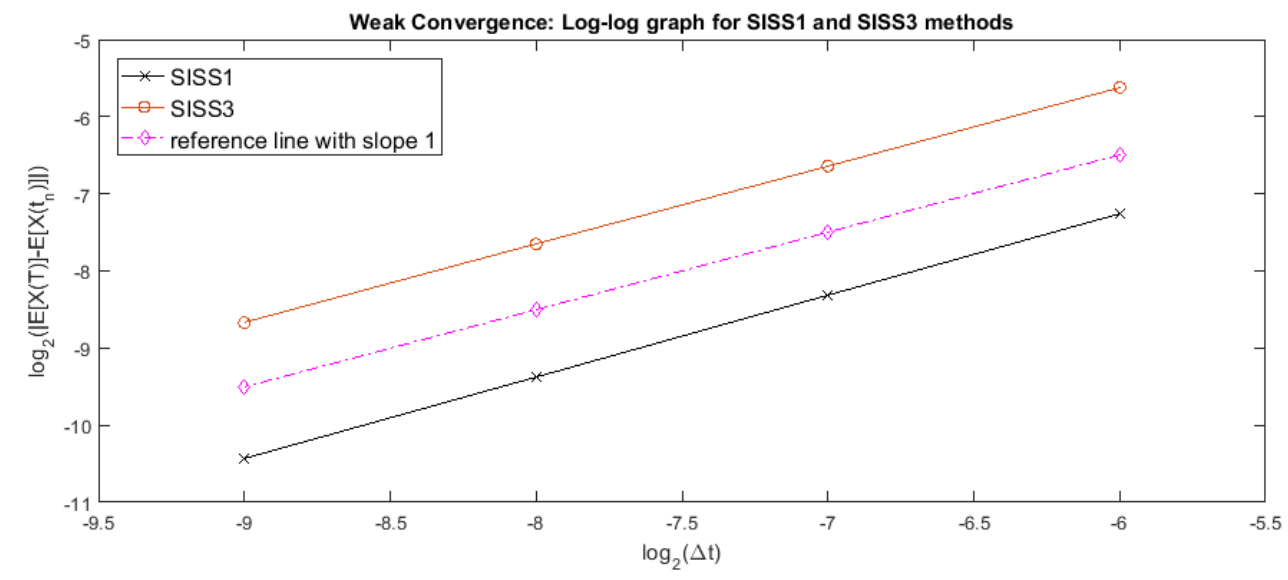

Fig. 4: Log-log graphs for the weak convergence rate of the SISS1 and SISS3 methods.

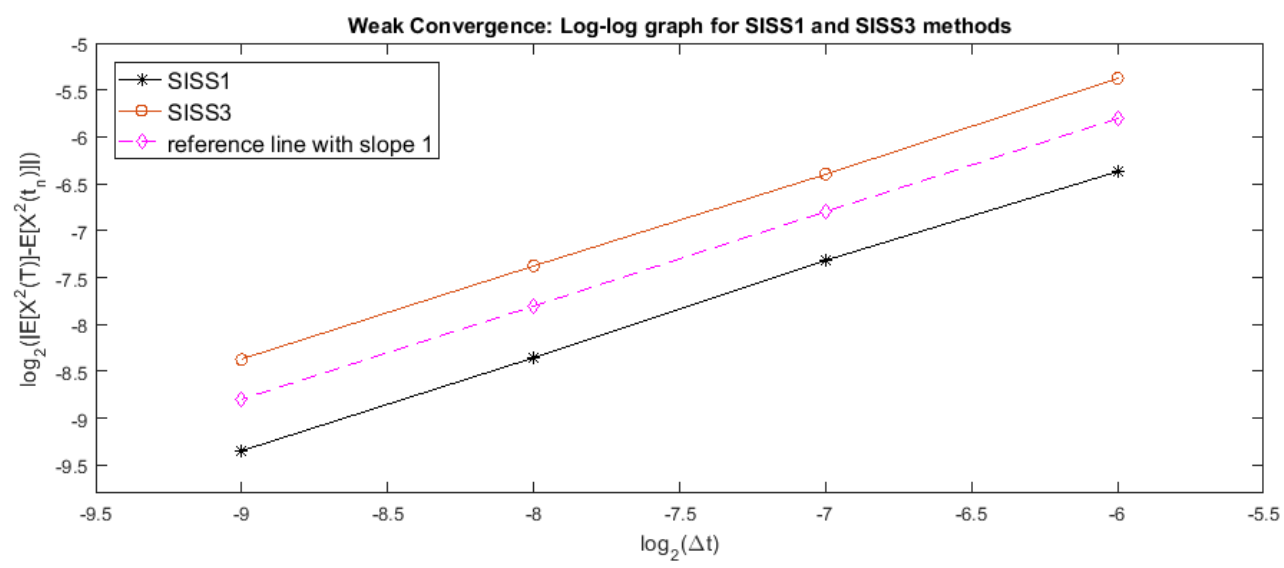

Fig. 5: Log-log graphs for the weak convergence rate of the SISS1 and SISS3 methods.

experiments show the robustness of the numerical solutions for the upper and lower boundaries of the first moment when $\Delta$ is relatively (or $n$ is big enough) small enough.

Author Contributions: All authors contributed equally to the writing of this paper. All authors read and approved the final manuscript.

Funding: This research received no external funding.

Conflicts of Interest: The authors declare no conflict of interest.

\section{References}

[1] M. Hutzenthaler, A. Jentzen and P.E. Kloeden. Strong and weak divergence in finite time of Euler's method for stochastic differential equations with non-globally Lipschitz continuous coefficients, Proceedings of the Royal Society, A, 467 (2130), pp. 1563-1576, 2011.

[2] M. Hutzenthaler, A. Jentzen and P.E. Kloeden. Strong convergence of an explicit numerical method for SDEs with non-globally Lipschitz continuous coefficients, Ann. Appl. Prob., 22, pp. 1611-1641, 2012. 
[3] S. Sabanis. A note on tamed Euler approximations, Electron. Commun. Prob. 18 (47), pp. 1-10, 2013.

[4] M. Hutzenthaler and A. Jentzen Numerical Approximations of nonlinear stochastic differential equations with non-globally Lipschitz continuous coefficients, Memoirs of the Amer. Math. Soc., 236, 1112 (second of 6 numbers), 2015.

[5] X. Mao. The truncated Euler-Maruyama method for stochastic differential equations, J. of Comp. and Appl. Math., 290, pp. 370-384, 2015

[6] X. Mao. Convergence rates of the truncated Euler-Maruyama method for stochastic differential equations, J. of Comp. and Appl. Math., 29p, pp. 362-375, 2016.

[7] J.C. Mattingly, A.M. Stuart and D.J. Higham. Ergodicity for SDEs and approximations: locally Lipschitz vector fields and degenerate noise, Stoch Proces. and Their Appl., 101, pp. 185-232, 2002.

[8] D. J. Higham, X. Mao and A. M. Stuart. Strong convergence of Euler type methods for nonlinear stochastic differential equations, SIAM J. Numer. Anal., 40 (3), pp. 1041-1063, 2002.

[9] H. Schurz. An axiomatic approach to numerical approximations of stochastic processes, Int. J. of Num. Analysis and Modeling, 3 (02), pp. 459-480, 2006.

[10] X. Mao and L. Szpruch. Strong convergence and stability of implicit numerical methods for stochastic differential equations with non-globally Lipschitz continuous coefficients, J. of Comp. and Appl. Math., 238, pp. 14-28, 2013.

[11] B. Izgi and C. Cetin. Some moment estimates for new semi-implicit split-step methods, AIP Conference Proceedings, Vol. 1833. No.1. AIP Publishing, 2017.

[12] B. Izgi and C. Cetin. Semi-implicit split-step numerical methods for a class of nonlinear SDEs with non-Lipschitz drift terms, J. of Comp. and Appl. Math., 343, pp. 62-79, 2018

[13] B. Izgi and C. Cetin. Milstein type semi-implicit split-step numerical methods for nonlinear SDE with locally lipschitz drift terms, Thermal Sciences, DOI: https://doi.org/10.2298/TSCI180912325I, 2018.

[14] B. Izgi and C. Cetin. Strong convergence of semi-implicit split-step methods for SDE with locally Lipschitz coefficients, Working paper, 2018.

[15] B. Izgi and C. Cetin. Strong convergence of Milstein-type semi-implicit split-step methods for SDEs with locally Lipschitz coefficients, Working paper, 2018.

[16] B. Izgi and B. Ari. Some Moment Boundaries and Simulation Results for the SISS Methods and Stochastic Ginzburg-Landau Equation, Working paper, 2019. 